Bio - grafía. Escritos sobre la Biología y su Enseñanza. ISSN 2027-1034

Edición Extraordinaria. p.p. 461 - 468

Memorias del IX Encuentro Nacional de Experiencias en Enseñanza de la Biología y la Educación Ambiental. IV Congreso Nacional de Investigación en Enseñanza de la Biología.

\title{
CONSTRUYENDO IDENTIDAD PROFESIONAL DOCENTE ENTRE MAESTROS DE CIENCIAS NATURALES
}

\section{BUILDING TEACHING PROFESSIONAL IDENTITY AMONG NATURAL SCIENCES TEACHERS}

\author{
Yesenia Quiceno Serna' \\ Ma. Mercedes Jiménez Narváez ${ }^{2}$ \\ Natalia Ramírez Agudelo ${ }^{3}$ \\ Adriana María Villegas Otálvaro ${ }^{4}$ \\ Adriana Marcela Torres Durán ${ }^{5}$
}

\section{RESUMEN:}

La presente comunicación incluye resultados parciales de una investigación ${ }^{6}$ que indaga sobre cómo una estrategia de trabajo colaborativo entre egresados y maestros en formación inicial de Ciencias Naturales, aporta en la construcción de su identidad profesional docente. Se buscan tres propósitos: la identificación de los elementos de la formación inicial que aportan a esta identidad, el reconocimiento de los saberes experienciales de los egresados que actúan como ejes de reflexión sobre su propia práctica y la valoración del diálogo entre pares, como contribución en el desarrollo profesional docente. Este trabajo, se enmarca en el paradigma cualitativo, privilegiando la estrategia de grupos de discusión. Desde esta perspectiva, se diseñaron talleres que tienen en cuenta los siete rasgos de la identidad profesional docente propuestos por Bolívar (2006); se busca que los participantes analicen aspectos concernientes a la labor docente desde sus experiencias personales y profesionales. Con ello, se aportarán insumos de reflexión para el Programa de formación inicial en el marco de la transformación curricular y principalmente, se espera potenciar el desarrollo profesional de los participantes.

\footnotetext{
${ }^{1}$ Magíster en Educación en Ciencias Naturales. Docente de cátedra, Universidad de Antioquia. Grupo PiEnCias. Correo: yesenia.quiceno@udea.edu.co

${ }^{2}$ Doctora en Educación. Profesora Asociada Facultad de Educación Universidad de Antioquia. Grupo PiEnCias. Correo: maria.jimenez@udea.edu.co

${ }_{3}^{3}$ Magíster en Educación en Ciencias Naturales. Docente de cátedra, Universidad de Antioquia. Grupo PiEnCias. Correo: natalia.ramirez2@udea.edu.co

4 Estudiante Maestría en Educación en Ciencias Naturales, Universidad de Antioquia. Grupo PiEnCias. Correo: adrianam.villegas@udea.edu.co

${ }^{5}$ Estudiante Doctorado en Educación. Facultad de Educación, Universidad de Antioquia. Grupo PiEnCias. Correo: amarcela.torres@udea.edu.co

6 Este trabajo es financiado por la Convocatoria de Permanencia con Equidad en la línea Egresados dinamizadores 2016, Vicerrectorías de Docencia y Extensión, Universidad de Antioquia.
} 
Bio - grafía. Escritos sobre la Biología y su Enseñanza. ISSN 2027-1034

Edición Extraordinaria. p.p. 461 - 468

Memorias del IX Encuentro Nacional de Experiencias en Enseñanza de la Biología y la

Educación Ambiental. IV Congreso Nacional de Investigación en Enseñanza de la Biología.

PALABRAS CLAVE: identidad profesional docente, formación inicial, práctica profesional, saber experiencial.

\section{ABSTRACT:}

This document includes the partial results of an investigation that explores how a collaborative work strategy among graduates and Pre-service Sciences Teachers contributes to the construction of their professional teaching identity. Three objectives are set: the identification of the elements of initial formation that contributes to this identity, the recognition of the experiential knowledge of the graduates that works as reflection axes on their own practice, and the acknowledge of the value of the dialogue among pairs, as a contribution to the Teacher Professional development. This work is framed in the qualitative paradigm, privileging the strategy of groups of discussion. From this perspective, workshops were designed taking into account the seven features of teacher professional identity proposed by Bolivar (2006); It is sought that participants analyze aspects concerning to teaching in the light of their personal and professional experiences. All this will provide reflection inputs for the program of initial training within the framework of curricular transformation, and mainly, it is hoped to enhance the professional development of the participants.

KEY WORDS: Professional teacher identity, pre-service teaching, in-service teacher education, experiential knowledge.

\section{INTRODUCCIÓN}

Esta investigación surge de la necesidad de reconocer los saberes profesionales de los maestros de ciencias naturales ${ }^{7}$ en ejercicio, que pueden dinamizar y permitir el avance en las reflexiones académicas y curriculares que requiere el programa de formación inicial del cual egresaron. De igual forma se sustenta en algunas situaciones que expresan los estudiantes de la Licenciatura frente al ingreso, permanencia y deserción, en tanto, por lo menos el 50\% de los admitidos han elegido el Programa por segunda opción, y algunos que han cancelado materias o el semestre, mencionan entre otras razones la dificultad de armonizar los horarios académicos con los laborales y la desorientación vocacional y profesional. Así mismo es preocupante que en algunos casos solamente al llegar al octavo semestre e iniciar las prácticas pedagógicas, los estudiantes comprendan cuál es su perfil profesional. Aunque la Licenciatura está en proceso de transformación y en la nueva versión se incluirán prácticas tempranas, los estudiantes que ya están transitando por ella no tendrán las mismas oportunidades de acercamiento a la realidad escolar y profesional.

\footnotetext{
${ }^{7}$ En todo el texto se hará referencia al Programa de Licenciatura en Educación Básica con énfasis en Ciencias Naturales y Educación Ambiental, Facultad de Educación, Universidad de Antioquia.
} 


\title{
Bio - grafía. Escritos sobre la Biología y su Enseñanza. ISSN 2027-1034
}

\section{Edición Extraordinaria. p.p. 461 - 468}

\author{
Memorias del IX Encuentro Nacional de Experiencias en Enseñanza de la Biología y la \\ Educación Ambiental. IV Congreso Nacional de Investigación en Enseñanza de la \\ Biología.
}

Desde el 2011, en la Licenciatura se han realizado algunos acercamientos a la temática de inserción profesional docente, que incluye el seguimiento a los egresados (Jiménez, Mejía y Montoya, 2012; Jiménez, 2013), la indagación por sus problemáticas como profesores principiantes (Ramírez, 2016) y se ha generado un dispositivo de acompañamiento a los mismos (Cividini, Jiménez, Mejía, y Morales 2016). Se ha identificado también que la formación inicial aporta elementos que influyen en la constitución de la identidad de los maestros en formación (Quiceno, 2016), pero al parecer éstos no han sido suficientemente tenidos en cuenta.

La temática de esta investigación se sustenta además en la revisión de literatura, que muestra una diversidad de estudios adelantados sobre el tema de identidad profesional docente (Cattonar, 2006; Flores, 2008; Pontes, Ariza y Del Rey, 2010; Domingo y Barrero, 2012; Delorenzi, Boubée y Nuñez, 2012) y sobre inserción profesional (Ávalos, Carlson, y Aylwin, 2004; Ávalos, 2009), y dan cuenta de la preocupación existente de las comunidades académicas por el sentimiento del maestro sobre sí mismo y su profesión. Escritos como el presentado por Vaillant (2007), ponen en evidencia cómo los cambios sociales generan problemas en la identidad profesional de los profesores, al contrastar sus expectativas personales acerca de la profesión y las realidades que caracterizan el trabajo docente en el aula de clase.

En consonancia con las ideas anteriores, algunos autores (Cattonar, 2006; Vaillant, 2007 y Ávalos, 2009) añaden que la constitución de la identidad profesional obedece a un proceso de naturaleza biográfica y relacional que depende en gran medida de la identidad propia y de aquella que fundamenta la sociedad sobre sí mismo como profesional. Además para comprender la identidad también se tiene en cuenta el conjunto de saberes teóricos y experienciales de la persona; Tardif (2004) considera "donde entran en juego elementos emocionales, de relación y simbólicos que permiten que un individuo se considere y viva como profesor y asuma así, subjetiva u objetivamente el hecho de realizar una carrera en la enseñanza" (p. 79).

De acuerdo con Bolívar (2006), la identidad profesional docente se puede comprender desde unos componentes que se yuxtaponen e interactúan, como son: la autoimagen, el reconocimiento social, el grado de satisfacción con el oficio, las relaciones sociales en el centro de trabajo, la actitud ante el cambio, las competencias profesionales y las expectativas futuras en la profesión; éstos se toman como categorías de análisis en el presente estudio.

Con referencia a las investigaciones que dan cuenta de la identidad profesional particular del profesor formado en Ciencias Naturales o que enseña Ciencias Naturales, se regis tran algunos trabajos en este campo en Estados Unidos (Volkmann y Anderson, 1998; Gilmore, Hurst y Maher, 2009; Siry y Lara, 2012 y Webb, 2012), España (Domingo y Pérez, 2005), Argentina (Delorenzi et al., 2012) y Colombia (Aristizábal y García, 2012). En estos estudios emergen algunos factores como son: motivación por la carrera, concepción de ciencia, visiones epistemológicas sobre la enseñanza y aprendizaje de las ciencias naturales, grado de dominio del saber disciplinar y finalidad de la educación científica -entre otros asuntos-; éstos entran a modelar la identidad que construyen los futuros profesores de ciencias naturales. Estos factores forman parte de su identidad, en 


\title{
Bio - grafía. Escritos sobre la Biología y su Enseñanza. ISSN 2027-1034
}

\section{Edición Extraordinaria. p.p. 461 - 468}

\author{
Memorias del IX Encuentro Nacional de Experiencias en Enseñanza de la Biología y la \\ Educación Ambiental. IV Congreso Nacional de Investigación en Enseñanza de la \\ Biología.
}

la medida en que su construcción proviene de los procesos de socialización -ya sea en la formación escolar y/o en la formación inicial- y de las vivencias que hacen parte de la biografía personal de los maestros.

Dada la ausencia de trabajos que den cuenta de esos elementos de identidad en la Licenciatura, y contando con la disposición que tienen los egresados por retornar a la Universidad así como la necesidad de aportar en la construcción del perfil profesional de los estudiantes, se genera esta propuesta de investigación. Entre las preguntas orientadoras se tienen: ¿Qué saberes surgen entre maestros en formación inicial y en formación continua cuando interactúan en un grupo de discusión?, ¿cómo ayudar en el reconocimiento de los saberes profesionales de los egresados y cómo éstos pueden aportar en la constitución de la identidad profesional de los maestros en formación?.

Este estudio busca aportar reflexiones y acciones no solo para los participantes directos de la estrategia, sino que aportará elementos académicos para las transformaciones que se esperan en los programas de formación inicial y continua de maestros de esta área.

\section{METODOLOGÍA}

El proyecto se enmarca en una perspectiva cualitativa, entendida como aquel tipo de investigación interesada en encontrar el sentido de un fenómeno social en su medio natural y desde una mirada holística (Filstead, 1986). Esta perspectiva favorece el propósito de investigación que se centra en el análisis de las vivencias que tienen los egresados y la manera en cómo sus experiencias al ser compartidas, discutidas y analizadas en conjunto con maestros en formación de la misma Licenciatura, aporta en la constitución de la identidad profesional individual y colectiva.

Se privilegió como técnica de investigación los grupos de discusión, entendiendo éstos como "cualquier debate de grupo en que el investigador estimule activamente la interacción del grupo y esté atento a ella" (Kitzinger y Barbour, 1999, citado por Barbour, 2013, p.25). Esta técnica se caracteriza por ser abierta y estructurada, donde el investigador, quien asume el rol de moderador, plantea preguntas o situaciones relacionadas al objeto de análisis, en torno a las cuales todos los participantes intervienen de forma libre, buscando aquellos puntos de consenso y disenso que tengan lugar (Bolívar, 2005).

En esta medida y dadas las características flexibles de este enfoque, se diseñaron siete sesiones del grupo de discusión mediante talleres, entendiendo éstos como dispositivos de formación y de socialización de las prácticas. Tal como lo menciona Hernández (2015) el taller conforma "un dispositivo de formación en el que se integran diferentes estrategias, con la intensión de abordar las tensiones propias entre la teoría y la práctica a partir de instancias de reflexión, posibilitando la ampliación, profundización y/o construcción de marcos referenciales" (p. 72). 


\title{
Bio - grafía. Escritos sobre la Biología y su Enseñanza. ISSN 2027-1034
}

\section{Edición Extraordinaria. p.p. 461 - 468}

\author{
Memorias del IX Encuentro Nacional de Experiencias en Enseñanza de la Biología y la \\ Educación Ambiental. IV Congreso Nacional de Investigación en Enseñanza de la \\ Biología.
}

El proyecto inició en octubre de 2016 y tendrá una duración de 12 meses. Se diseñaron y desarrollaron 7 sesiones de encuentro entre los participantes, las cuales fueron grabadas en audio y video, para luego ser transcritas, e iniciar el análisis de las mismas.

Los participantes son egresados y maestros en formación de la misma Licenciatura que voluntariamente aceptaron asistir; así mismo, el equipo de investigación que está conformada por profesoras y una joven investigadora (estudiante de ciencias naturales), quienes diseñan y orientan el proceso.

Para la conformación del grupo de participantes se utilizaron las bases de datos de estudiantes y egresados de la Licenciatura. Se realizó una convocatoria abierta de preinscripción por medio electrónico; posteriormente se definieron las fechas y horarios de encuentro (sábados 9:00 a 12:00); los encuentros se realizaron cada 20 días o un mes, entre abril y agosto del año en curso. Para formalizar la participación de los estudiantes y egresados, en el primer encuentro se socializaron las características de la investigación y se suscribieron los consentimientos del proceso (Hutchinson y Wilson, 2003) definiendo los compromisos éticos de confidencialidad y autorización de uso de la información.

\section{AVANCES DE LAINVESTIGACIÓN:}

A la fecha de elaboración de esta comunicación, ya se realizaron las 7 sesiones donde participaron en promedio 15 maestros. Las actividades propuestas se concentraron principalmente en los rasgos de identidad profesional correspondientes a: la autoimagen, el reconocimiento social, relaciones con los colegas, la actitud frente al cambio y expectativas de futuro en la profesión; las competencias profesionales y el grado de satisfacción se integran como ejes transversales. Se realizaron actividades individuales y en grupos, algunas de ellas: dramatizaciones, lectura de textos, construcción de línea del tiempo, el análisis de casos y de diversos productos narrativos.

La revisión preliminar de la información recogida muestra que los participantes valoran positivamente la estrategia de interacción entre egresados y estudiantes. Por un lado, los egresados resaltan la posibilidad de retornar a la Universidad a un espacio de diálogo, escucha y de respeto por la palabra, donde las expresiones no quedan en lo anecdótico y la queja, sino que trascienden a la reflexión personal y profesional. Por otro lado, los estudiantes valoran la posibilidad de escuchar de fuente directa las funciones que cumple un profesor de ciencias, las actividades que puede realizar, las relaciones que establece en la comunidad académica, los ámbitos en los que puede trabajar (educativo formal, no formal).

Ambos grupos reconocen y comparten los temores y retos que tiene la profesión, así como algunos de factores que influyen en la carrera docente en el país, por ejemplo, los procesos de ingreso y evaluación docente. Para finalizar este avance, dado que el análisis apenas está en construcción, incluimos una expresión de una de las participantes, con la cual podemos afimar que esta experiencia de formación alcanzó los objetivos esperados frente a la construcción de la identidad profesional docente: 


\section{Bio - grafía. Escritos sobre la Biología y su Enseñanza. ISSN 2027-1034}

Edición Extraordinaria. p.p. 461 - 468

Memorias del IX Encuentro Nacional de Experiencias en Enseñanza de la Biología y la

Educación Ambiental. IV Congreso Nacional de Investigación en Enseñanza de la Biología.

lo más importante fue conocer otras personas con situaciones profesionales muy similares a las mías, darme cuenta que lo que me ha pasado como profesora no solo lo he vivido yo, y sobretodo escuchar docentes con más experiencia contando como podemos enfrentar esas situaciones complejas. Aprendí a tener mi mente más abierta a todo lo que puede suceder en mi vida profesional, aprendí que los temores pueden ser una herramienta para afrontar las dificultades, aprendí que es mi actitud ante la vida la que me permitirá salir adelante, aprendí que la construcción de mi identidad como docente es un proceso en constante cambio que me exige una transformación constante (Estudiante; Evaluación final; S.7).

\section{BIBLIOGRAFÍA}

Aristizábal, A., \& García, A. (2012) Construcción de la identidad profesional docente: ¿posibilidad o utopía?. Revista EDUCyT, Extraordinario, Diciembre.

Ávalos, B., Carlson, B., \& Aylwin, P. (2004). La inserción de profesores neófitos en el Sistema educativo: ¿cuánto sienten que saben y cómo perciben su capacidad docente en relación con las tareas de enseñanza asignadas? Concurso Nacional de Proyectos Fondecyt Regular 2002 No. 1020218. Santiago, Chile.

Ávalos, B. (2009). La inserción profesional de los docentes. Profesorado. Revista de Currículum y Formación de Profesorado,13(1), 43-59.

Barbour, R. (2013). Los grupos de discusión en Investigación Cualitativa. Madrid: Morata.

Bolívar, A. (2006). La identidad profesional del profesorado de Secundaria: crisis y reconstrucción. Archidona (Málaga): Aljibe.

Bolívar, A., (2005). Conocimiento didáctico del contenido y didácticas específicas. Profesorado. [versión electrónica]. Revista de currículum y formación del profesorado, 9(2). Extraído 10 julio 2008 en, http://www.ugr.es/ recfpro/Rev92.html

Cattonar, B. (2006). Convergence et diversité de l'identité professionnelle des enseignantes et des enseignants du secondaire en communauté française de Belgique: Tensions entre le vrai travail et le sale boulot. Éducation et Francophonie, 34(1), 193-212.

Cividini, M., Jiménez, M., Mejía, L. \& Morales, A. (2016). "Y llega uno y se estrella con un montón de cosas": Saberes y problemas de profesores principiantes de ciencias naturales. Memorias $\mathrm{V}$ congreso internacional sobre el profesorado principiante y la inducción a la docencia. República Dominicana, 6-8 junio. Extraído el 2 de junio 2017 de: http://congressoprinc.com.br/artigo?id artigo $=636$

Delorenzi, O., Boubée, C., \& Nuñez, R. (2012). Las concepciones sobre la naturaleza de la ciencia: Incidencia en la construcción de la identidad en las primeras inserciones laborales. En III Jornadas de Enseñanza e Investigación Educativa en el campo de 


\section{Bio - grafía. Escritos sobre la Biología y su Enseñanza. ISSN 2027-1034}

Edición Extraordinaria. p.p. 461 - 468

Memorias del IX Encuentro Nacional de Experiencias en Enseñanza de la Biología y la Educación Ambiental. IV Congreso Nacional de Investigación en Enseñanza de la Biología.

las Ciencias Exactas y Naturales. Facultad de Humanidades y Ciencias de la Educación. Universidad Nacional de la Plata. Buenos Aires, Argentina.

Deslauriers, J. P., (2004). Investigación cualitativa. Guía Práctica. Versión al español y edición al cuidado de Miguel Ángel Gómez. Pereira: Ed. Papiro.

Domingo, J., \& Pérez, P. (2005). Crisis de identidad profesional y formación del profesorado de ciencias en la ESO. Enseñanza de las ciencias, Número extra. VII Congreso 1-4.

Domingo, J., \& Barrero, B. (2012). Reconstrucción de la identidad profesional del profesorado. Una mirada desde la orientación y la dirección. Revista Perspectiva Educacional, 51(2) 91-118.

Gilmore, J., Hurst, M., \& Maher, M. (2009). Professional identity development in teachers of science, technology, engineering, math, and science and math education. Proceedings of the NARST 2009 Annual Meeting. Extraído de: http://www.eric.ed.gov/PDFS/ED509408.pdf

Filstead, W., (1986). Una experiencia necesaria en la investigación evaluativa. En T., Cook, y CH. Reichart, Métodos cualitativos y cuantitativos en investigación evaluativa [pp.59-76]. Madrid: Ediciones Morata.

Flores, M. (2008). La investigación sobre los primeros años de enseñanza: lecturas e implicaciones. C. Marcelo (Coord.) El profesor principiante. Inserción a la docencia. (pp. 59 - 98). Barcelona: Ediciones octaedro.

Hernández, A. (2015). El taller como dispositivo de formación y socialización de las prácticas. En L. Sanjurjo (Ed). Los dispositivos para la formación en las prácticas profesionales [pp. 71-106]. Rosario, Argentina: Homo Sapiens Ediciones. 4ta Reimpresión.

Hutchinson, S. \& Wilson, H., (2003). La investigación y las entrevistas terapéuticas: una perspectiva postestructuralista. En J. Morse, (Edit.). Asuntos críticos en los métodos de investigación cualitativa. (pp. 349 -366) 1ra ed. en español. Medellín: Editorial Universidad de Antioquia.

Jiménez, M.M. (2013). La configuración del conocimiento del profesor principiante de ciencias naturales, en la inserción profesional. Tesis Doctoral. Facultad de Educación. Universidad de Antioquia: Medellín (sin publicar).

Jiménez, M.M., Mejía, L.S. \& Montoya, L.C., (2012). Profesores y profesoras de ciencias naturales y educación ambiental: problemas que enfrentan en su ejercicio profesional. Memorias III Congreso Internacional sobre Profesorado Principiante e Inserción Profesional a la Docencia. Santiago de Chile, 29 de febrero - 2 de marzo. Universidad de Sevilla y Universidad Autónoma de Chile. Apoyo de OEI y Asociación Chilena de Municipalidades. CD-ROM.

Pontes, A., Ariza, L., \& Del Rey, R. (2010). Identidad profesional docente en aspirantes a 
Bio - grafía. Escritos sobre la Biología y su Enseñanza. ISSN 2027-1034

Edición Extraordinaria. p.p. 461 - 468

Memorias del IX Encuentro Nacional de Experiencias en Enseñanza de la Biología y la

Educación Ambiental. IV Congreso Nacional de Investigación en Enseñanza de la Biología.

profesorado de enseñanza secundaria. Psychology, Society, \& Education, 2(2), 131142.

Ramírez, N. (2016). El proceso de inserción profesional del profesor principiante de Ciencias Naturales: Cuatro casos en Colombia. Trabajo de Maestría. Facultad de Educación, Universidad de Antioquia. Extraído de: http://ayura.udea.edu.co:8080/ispui/bitstream/123456789/2295/1/JE0856 nataliaram irez insercionprofesional.pdf

Quiceno, Y. (2016). (Re) constitución de la identidad profesional del profesor principiante de ciencias: una visión desde sus saberes en la etapa de inserción a la docencia. Trabajo de Maestría. Facultad de Educación, Universidad de Antioquia.

Siry, C., \& Lara, J. (2012). "I didn't know water could be so messy": coteaching in elementary teacher education and the production of identity for a new teacher of science. Cultural Studies of Science Education, 7(1), 1-30.

Vaillant, D. (2007). La identidad docente. I Congreso Internacional "Nuevas Tendencias en la Formación Permanente del Profesorado". PREAL. Barcelona.

Volkmann, M., \& Anderson, M. (1998). Creating professional identity: Dilemmas and metaphors of a first-year chemistry teacher. Science Education, 82(3), 293-310.

Webb, A. W. (2012). " Supporting" Beginning Secondary Science Teachers through Induction: A Multi-Case Study of Their Meaning Making and Identities. (Disertación Doctoral, University of North Carolina at Greensboro,2012). Dissertation ERIC. 